\title{
Early Surgery in Inflammatory Bowel Diseases Is a Better Option than Prolonged Conservative Treatment
}

\author{
Anton J. Kroesen \\ Allgemein- und Viszeralchirurgie, Krankenhaus Porz am Rhein, Cologne, Germany
}

Keywords

Ileoanal pouch $\cdot$ Intraepithelial neoplasia $\cdot$ Colorectal carcinoma $\cdot$ Fistula $\cdot$ Stenosis $\cdot$ Inflammatory bowel diseases

\section{Abstract}

Early surgery is a very important aspect of treatment of inflammatory bowel diseases. In Crohn's disease, early surgery should be performed in emergencies, in refractory courses, and in special cases at the beginning of the disease if there is a stenosis limited to the terminal ileum. In ulcerative colitis, prolonged therapy with extended application of all available substances should be avoided. Every therapy with more than 2 biologicals endangers the patient. Low-grade intraepithelial neoplasia (IEN) should also be resected earlier due to a $23 \%$ risk of synchronous and metachronous highgrade IEN or cancer.

(c) 2019 S. Karger AG, Basel

Although many new antibodies with many new targets have become available during the last two decades, there is no breakthrough for inflammatory bowel diseases in sight. Even the most modern antibodies have sustainable remission rates of more than $40 \%$ [1]. Unfortunately, due to the fear of patients and gastroenterologists, the indication for surgery is set by far too low. This article wants to illustrate the most crucial aspects in both Crohn's disease and ulcerative colitis.

\section{KARGER}

(0) 2019 S. Karger AG, Basel

\section{Early Surgery in Crohn's Disease}

\section{Emergency}

The clearest situation is the emergency situation. In Crohn's disease, we have the following indications:

\section{Small Bowel Obstruction due to Stenosis}

This indication can be ambivalent. In most of the cases, the patient can be helped in this situation with a high dose of prednisone over a short period of time (e.g., 100 mg daily for 2-3 days). This treatment leaves an operation at a later time point as an option. Nevertheless, it is necessary to observe the patient very thoroughly, since corticosteroids can ameliorate the symptoms of small bowel obstruction. In this case, emergency surgery is indicated. But a resection of the Crohn-affect bowel segment should also be performed. In case the remaining pre-stenotic bowel is too dilated, a diversion as a split stoma of the bowel ends should be made. The restoration of the continuity of the bowel can then be done after 2-3 months.

\section{Perforation}

There is no doubt that a perforation is an indication for very urgent surgery. As soon as clinical examination, $\mathrm{X}$-ray of the abdomen, or CT scan has confirmed the diagnosis, an emergency laparoscopic laparotomy should be performed with the aim to resect the perforation in-

Prof. Dr. med. Anton J. Kroesen

Allgemein- und Viszeralchirurgie, Krankenhaus Porz am Rhein Urbacher Weg 19

DE-51149 Cologne (Germany)

E-Mail a.kroesen@khporz.de 
cluding the Crohn-wearing bowel segment. Oversewing of the perforation is contraindicated and a crucial therapeutic mistake. Depending on the severity of the peritonitis, an anastomosis should only be constructed in very early perforations. Otherwise, a diversion of the two bowel ends as a common enterostomy is the better option.

Toxic Course in Crohn's Colitis/Refractory Bleeding

This condition is very often overlooked in Crohn's disease. In the same way as toxic colitis in ulcerative colitis can happen, this is also possible in Crohn's colitis. In this case and with a short history of the disease, a conservative treatment can be performed using high-dose corticoids or calcineurin inhibitors. Anti-TNF- $\alpha$ antibodies are basically also an option. But in cases where emergency surgery becomes necessary quickly (2-7 days), the patient then has to be operated on under full anti-TNF- $\alpha$ inhibition with a high risk of nonsurgical complication such as infections and intra-abdominal abscesses, whereas calcineurin inhibitors work only for $48 \mathrm{~h}$.

A patient who has a prior history of a toxic course should be operated immediately since the success of a conservative treatment is even less likely in such a situation. Refractory bleeding consuming more than 4 units of blood per $24 \mathrm{~h}$ is always an indication for surgery. In both cases (toxic course and refractory bleeding), a colectomy with rectal stump closure and ileostomy should be done.

\section{Early Surgery in lleitis Terminalis at the Beginning of} Crohn's Disease

Several medical publications reported a lower recurrence rate at the beginning of Crohn's disease $[2,3]$. These two publications led to a randomized trial designed by a Dutch group of scientists (LIric trial). Based on a multicenter trial including 29 hospitals, this study compared 70 patients who received infliximab at the beginning of their disease history with 73 patients who were operated using ileocecal resection also at the beginning of their disease history. After 7-18 months, $84 \%$ of the patients in the infliximab group were in remission, whereas $79 \%$ of the patients treated by ileocecal resection were in remission. The authors compared many categories of quality of life using the IBDQ score and could not show any significant differences between the two groups [4]. However, after 27 weeks, $20 \%$ of the patients initially treated with infliximab underwent surgery, whereas only $2.6 \%$ of the resected patients received infliximab. In view of the fact that the authors performed an intentionto-treat analysis, one can conclude that patients with stricturizing Crohn's disease limited to the terminal ileum are better treated by ileocecal resection, which is a clear plea for early surgery.

\section{Early Surgery to Prevent Superfluous Medical \\ Treatment}

The fact that the number of medical treatment options has increased tremendously seduces many gastroenterologists and patients to try "everything." However, as soon as a simultaneous continuous corticosteroid medication has to be prescribed or a third-line biological treatment should be started, surgery is the better option as long as the patient is operable (in view of the danger of short bowel syndrome and functional operability).

\section{Ulcerative Colitis}

Also in ulcerative colitis, we see emergencies in about $4 \%$ of all cases as an indication for early surgery. These indications are: a toxic course (very rare with megacolon), refractory bleeding, perforation, and bowel obstruction. In patients with a toxic course and refractory bleeding, the same procedure as that already described for Crohn's colitis should be followed: a toxic course is a life-threatening emergency situation. When all conservative options have been tried, the only possibility for an actual therapy is a colectomy with arrangement of an end-standing ileostomy and Hartmann's procedure or sigmoid mucous fistula. The attempt of a long-lasting conservative therapy must be avoided on account of the still high mortality. In the acute phase, surgeon and gastroenterologist must stand side by side at the patient's bed and decide when to operate.

Patients with a short course of their disease can be treated more extensively than patients who have already had a toxic course in their history. Anti-TNF- $\alpha$ antibodies should be avoided due to their long half-time, although they are effective in this situation.

\section{Bleeding}

If bleeding lasts for $24 \mathrm{~h}$ consuming more than 4 units of blood, colectomy is indicated. The same surgical strategy should be performed as in a toxic course.

\section{Early Surgery in Intraepithelial Neoplasia}

After 10 years of illness, the number of colitis carcinomas rises up to $1 \%$, after 30 years of illness, this figure amounts to $17 \%$. In case of a pancolitis, the risk of neoplasia raises up to $14.8 \%$. The carcinomas can appear in all segments of the colon [5]. Furthermore, the only predictor of ulcerative colitis-related colon carcinoma is intraepithelial neoplasia (IEN). Hence, in all high-risk pa- 
tients (illness $>10$ years; pancolitis), an annual colonoscopy with multiple biopsies should be carried out.

The relative indication for a restorative proctocolectomy exists with high-grade IEN, while with high-grade IEN, an absolute indication for an operation is given. Very recently, new evidence has been published by a Dutch group [6]. This publication reports that $23 \%$ of all colectomies for low-grade IEN showed synchronous or metachronous high-grade neoplasia or carcinoma. In view of this new evidence, the indication for surgery also in low-grade IEN should be set earlier [6].

\section{Therapy-Refractory Course}

A refractory course in ulcerative colitis is the predominant indication for surgery. About $80 \%$ of all patients are operated on for this reason. Until now, 4 basic therapeutics exist (prednisolone, mesalamine, azathioprine, sulfasalazine), 2 calcineurine inhibitors (tacrolimus, cyclosporine), at least 4 anti-TNF- $\alpha$ antibodies, 1 anti-integrin antibody, 1 IL-12/23 antibody, and 1 JAK inhibitor. None of these medications has a persistent remission rate of more than $40 \%$, and the publication by Singh et al. [7] has shown that the response rate to a second-line therapy with anti-integrin, anti-IL, or JAK inhibitors is $<24 \%$. Every other antibody has a response rate $<30 \%$. Hence, the chance to save a patient with a second- or third-line or even higher number-line therapy is very small. But on the other hand, the risk of disease progression is $>70 \%$. Every patient who is treated with one of these options faces the risk of disease progression and quality of life deterioration.

In view of this, also patients with a therapy-refractory course of ulcerative colitis should be operated early. At

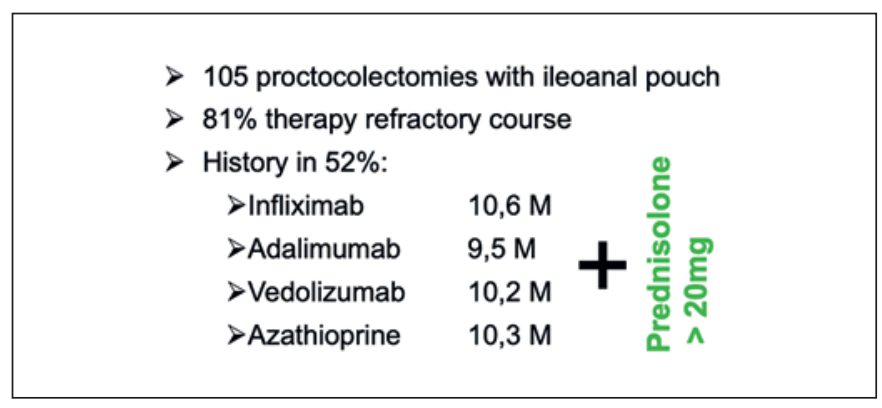

Fig. 1. Analysis of 105 consecutive proctocolectomies with ileoanal pouch in the year 2018 and their preoperative medications.

the latest after 2 antibody therapies without a significant change in the severity of the colitis, surgery is the better option. This claim counts even more after analyzing the facts. A review of all proctocolectomies of the last year at our institute found that $52 \%$ of all patients had 4 biologicals plus uninterrupted administration of more than 20 $\mathrm{mg}$ of prednisolone (Fig. 1). All of these patients are at a higher risk of surgery and should have been operated much earlier with a probably lower risk for complications.

Surgery helps in $100 \%$ of all cases, it has a quite high morbidity rate of $20-30 \%$, about $50 \%$ of all patients experience a - mostly singular - episode of pouchitis, and mortality is $0.8 \%$, but on the other hand, $90 \%$ of all patients have a long-time success rate with a tremendous improvement of their quality of life [8].

\section{Disclosure Statement}

The author declares no conflicts of interest.

\section{References}

1 Damião AO, de Azevedo MF, Carlos AS, Wada MY, Silva TV, Feitosa FC. Conventional therapy for moderate to severe inflammatory bowel disease: A systematic literature review. World J Gastroenterol. 2019 Mar;25(9): 1142-57.

2 Aratari A, Papi C, Leandro G, Viscido A, Capurso L, Caprilli R. Early versus late surgery for ileo-caecal Crohn's disease. Aliment Pharmacol Ther. 2007 Nov;26(10):1303-12.

3 Latella G, Cocco A, Angelucci E, Viscido A Bacci S, Necozione S, et al. Clinical course of Crohn's disease first diagnosed at surgery for acute abdomen. Dig Liver Dis. 2009 Apr; 41(4):269-76
4 Fazio VW, Kiran RP, Remzi FH, Coffey JC, Heneghan HM, Kirat HT, et al. Ileal pouch anal anastomosis: analysis of outcome and quality of life in 3707 patients. Ann Surg. 2013 Apr;257(4):679-85.

5 Ponsioen CY, de Groof EJ, Eshuis EJ, Gardenbroek TJ, Bossuyt PM, Hart A, et al.; LIR!C study group. Laparoscopic ileocaecal resection versus infliximab for terminal ileitis in Crohn's disease: a randomised controlled, open-label, multicentre trial. Lancet Gastroenterol Hepatol. 2017 Nov;2(11):785-92.

6 de Jong ME, van Tilburg SB, Nissen LH, Kievit W, Nagtegaal ID, Horjus CS, et al. Longterm risk of advanced neoplasia after colonic low-grade dysplasia in patients with inflammatory bowel disease: a nationwide cohort study. J Crohn's Colitis. 2019 Jun;jzz114.
7 Singh S, Fumery M, Sandborn WJ, Murad MH. Systematic review with network metaanalysis: first- and second-line pharmacotherapy for moderate-severe ulcerative colitis. Aliment Pharmacol Ther. 2018 Jan;47(2): 162-75.

8 Eaden J, Abrams K, Ekbom A, Jackson E, Mayberry J. Colorectal cancer prevention in ulcerative colitis: a case-control study. Aliment Pharmacol Ther. 2000 Feb;14(2):14553. 